\title{
ESTUDO DO EFEITO DO COEFICIENTE DE ESPECULARIDADE NA SIMULAÇÃO FLUIDODINÂMICA DE UM SECADOR DE LEITO DESLIZANTE COM ESCOAMENTO CONTRACORRENTE
}

\author{
A. A. PFEIFER ${ }^{1 *}$, M. A. S. BARROZO ${ }^{2}$, L. D. SILVA ${ }^{1}$ \\ ${ }^{1}$ Universidade Federal do Triângulo Mineiro, Departamento de Engenharia Química \\ ${ }^{2}$ Universidade Federal de Uberlândia, Faculdade de Engenharia Química \\ *e-mail: adriene_br@yahoo.com.br
}

\begin{abstract}
RESUMO
Este trabalho buscou através da técnica da simulação fluidodinâmica estudar o comportamento das partículas (sementes de soja) dentro de um secador de leito deslizante, de forma a compreender melhor o efeito de parede e obter perfis de porosidade e velocidade ao longo do leito deslizante. Para tal, estudou-se o efeito da variação do coeficiente de especularidade em um secador de leito deslizante com escoamento contracorrente, com diâmetro de $0,081 \mathrm{~m}$ e comprimento de $0,528 \mathrm{~m}$. Para o estudo do perfil de porosidade e velocidade, foram analisados os resultados das simulações para o tempo de 10 segundos, com velocidade de entrada do ar no secador igual a $1,42 \mathrm{~m} \cdot \mathrm{s}^{-1}$, variando o coeficiente de especularidade de 0 a 1 . Pode ser observado, para linhas de análise diversas (região superior da entrada de ar do secador, região central do leito e região da saída do ar de secagem) que há uma variação do perfil de porosidade com a aproximação da parede do secador; sendo que, com o aumento do coeficiente de especularidade o perfil se aproxima do perfil de porosidade sem este coeficiente e que o perfil se torna amortecido na região central do leito. Os resultados demonstraram que para a situação em que a célula encontra-se com sólidos em escoamento, as regiões próximas à parede do secador apresentam níveis superiores de velocidade, comprovando que há um perfil de porosidade e de velocidade do ar de secagem em um secador de leito deslizante e que este não é plano.
\end{abstract}

\section{INTRODUÇÃ̃O}

A produção de soja tem grande expressão na economia e na cultura agrícola brasileira, visto seu alto teor de óleo e proteína que geram inúmeros produtos $\mathrm{e}$ subprodutos, além de que o seu cultivo gera postos de trabalho no campo, diversos benefícios à região produtora e a movimentação do mercado agroindustrial.

A manutenção da qualidade da soja para o replantio (sementes) é fator indispensável; sendo utilizado para isso o método de secagem. $O$ processo de secagem pode ser realizado de modo natural ou artificial. A secagem artificial é realizada em secadores, nos quais as sementes são submetidas a um fluxo de ar que pode ser aquecido ou não, o que promove a secagem num período de tempo relativamente curto. Entre os secadores industriais, o secador de leito deslizante tem apresentado algumas vantagens em relação aos demais, tais como: menor investimento e baixo consumo de energia, além de provocar menos injúrias mecânicas às sementes (BARROZO, 1995). As três configurações clássicas são definidas de acordo com a direção de escoamento do fluido em relação 
ao sólido, podendo ser concorrentes, contracorrentes e cruzados.

$\mathrm{Na}$ tentativa de obter informações necessárias ao entendimento dos fenômenos que ocorrem no leito deslizante pode-se realizar a simulação fluidodinâmica deste sistema, que corresponde a uma técnica utilizada para estudar a dinâmica dos fluidos através de programas e metodologias de análise numérica. Os códigos de fluidodinâmica computacional comerciais (CFD, do inglês, Computational Fluid Dynamics) são resolvedores de sistemas de equações que se baseiam nos princípios de conservação de massa, energia e quantidade de movimento. Estes códigos propiciam a geração de malhas computacionais, o controle e o acompanhamento da solução no decorrer das iterações.

A utilização desta metodologia, com utilização de simuladores visa fornecer informações para os modelos matemáticos constituídos de equações de balanço de massa e energia, nas quais a resolução destes sistemas baseia-se em hipóteses simplificadoras, como perfil plano de velocidade.

Desta forma, o objetivo deste trabalho foi estudar o efeito do coeficiente de especularidade (specularity coefficient) na simulação fluidodinâmica de um secador de leito deslizante com escoamento contracorrente.

\section{REVISÃO BIBLIOGRÁFICA}

Os tipos de secadores utilizados na secagem de sementes podem ser divididos de acordo com o leito, em secadores de leito fixo, fluidizado e secadores de leito deslizante. Sendo que todas as configurações trabalham com a circulação de ar. Entre os secadores industriais, os secadores do tipo de leito deslizante apresentam menor investimento, baixo consumo energético e menores danos aos grãos e sementes (BARROZO, 1995).

Os secadores ainda são classificados em relação às direções dos escoamentos de sólidos e de fluido, sendo concorrentes, contracorrente e cruzados. $\mathrm{Na}$ secagem com escoamento contracorrente, as fases sólida e fluida, apresentam escoamentos paralelos, porém em sentidos opostos. Nesta configuração, o produto sai do secador com temperatura alta, próxima da temperatura de entrada do fluido. Entretanto, é a configuração em que se verificam as maiores taxas de redução no teor de umidade dos sólidos (VALENÇA e MASSARANI, 1996).

Nos estudos de simulação dos balanços de massa e energia do sistema de secagem de sementes de soja em leito deslizante presentes na literatura, observa-se poucos estudos referentes à análise dos perfis de porosidade $\mathrm{e}$ de velocidade dentro de um leito preenchido com o material a ser seco. São encontrados poucos trabalhos que relacionam o efeito de parede ao modelo matemático da secagem (BÉTTEGA, CORRÊA e FREIRE, 2007; LIRA, BARROZO e ASSIS, 2006, 2009; PFEIFER, 2011); porém autores como Vortmeyer e Schuster (1983), Fahien e Stankovich (1979), Souza (2001), Pfeifer (2011) entre outros, observaram que existem diferenças significativas nos valores das velocidades do ar ao longo do diâmetro do leito empacotado, desconsiderando a hipótese de que o perfil de velocidade é plano.

A Fluidodinâmica Computacional consiste na solução da equação geral de transporte empregando métodos numéricos, como o Método dos Volumes Finitos e o Método dos Elementos Finitos, visando obter campos de velocidades, temperatura etc., possibilitando avaliar o transporte de uma propriedade de interesse. Tal ferramenta apresenta grande interesse do ponto de vista da Engenharia Química, pois a maioria dos processos desta área envolve mais de uma fase, o que acarreta uma dependência da 
transferência de massa e reação química com a concentração local e as superfícies de transferência locais, interfaces gás/líquido, gás/partícula (PFEIFER, 2011).

Os códigos CFD comerciais são resolvedores de sistemas de equações que baseiam-se nos princípios de conservação de massa, energia e quantidade de movimento. Estes códigos oferecem recurso para a confecção da malha, e permite o acompanhamento e controle das iterações no decorrer da solução.

O coeficiente de especularidade descrito no software Fluent ${ }^{\circledR}$ (Fluent, 2006), é uma medida da fração de colisões que transferem movimento à parede e seu valor permanece entre zero e 1. Quando o valor é zero, esta condição equivale ao zero do coeficiente de cisalhamento da parede, mas quando é próximo da unidade, há uma grande quantidade de transferência de movimento (PFEIFER, 2011).

Diante do exposto, busca-se através da técnica de fluidodinâmica computacional estudar a dinâmica dos fluidos através de análises numéricas e observar o comportamento das partículas (sementes de soja) dentro do leito do secador de leito deslizante, de forma a compreender melhor o efeito de parede e obter perfis de porosidade e velocidade ao longo do leito deslizante.

\section{METODOLOGIA}

Para a simulação numérica, o secador foi dividido em pequenos volumes de controle, nos quais foram aplicadas as equações de transporte. A malha bidimensional, com aproximadamente 36.000 células quadrilaterais, criada para representar esse equipamento foi desenvolvida no

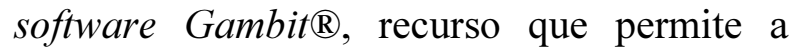
criação de malhas computacionais com compatibilidade para o resolvedor numérico Fluent ${ }^{\circledR} 14$.
As etapas de construção da malha foram elaboradas, conforme as etapas descritas por Duarte (2006):

- Construção da geometria do equipamento;

- Definição das faces e, ou volumes;

- Determinação de efeitos de camada limite ou outro tipo de refinamento desejado;

- Aplicação da malha no corpo geométrico construído e determinação do tipo e tamanho das células (quadrangular, tetraédrica, hexaédrica ou híbrida);

- Definição das paredes, interiores, entradas e saídas do equipamento;

- Determinação das fases que compõem o interior do equipamento - fluido (ar) e/ou sólido (sementes de soja);

- Conversão do arquivo contendo a malha em uma extensão reconhecida pelo software Fluent ${ }^{\circledR} 14$.

A figura 1 apresenta o esquema do secador de leito deslizante utilizado neste trabalho. A vazão de sólidos na saída das sementes é controlada por uma esteira, distante $0,006 \mathrm{~m}$ da saída do secador, com velocidade de $1,0.10^{-5} \mathrm{~m} \cdot \mathrm{s}^{-1}$. O diâmetro do secador é $0,081 \mathrm{~m}$, com comprimento de $0,528 \mathrm{~m}$. A escolha desta configuração foi feita a partir de dados de Souza (2011), que realizou estudos experimentais em uma unidade de secagem. Buscou-se representar o mesmo leito para fins de comparações.

A malha gerada foi, em seguida, caracterizada conforme as condições de operação do equipamento, os modelos, a solução numérica adotada e os critérios de convergência.

Neste trabalho foi utilizado o software Fluent ${ }^{\circledR} 14$ para as simulações, considerando regime transiente, aproximação do tipo EulerEuler e aplicou-se o modelo Euleriano. O tratamento do acoplamento entre a pressão e a velocidade foi feito por meio do algoritmo SIMPLE. Para as interpolações da equação do movimento e das frações de volume foi utilizado o esquema Upwind de primeira 
ordem. Utilizou-se um passo de tempo de $1,0.10^{-6} \mathrm{~s}$ e convergência de $1,0.10^{-4}$.

Figura 1 - Esquema do secador de leito deslizante com escoamento contracorrente com descrição das regiões.

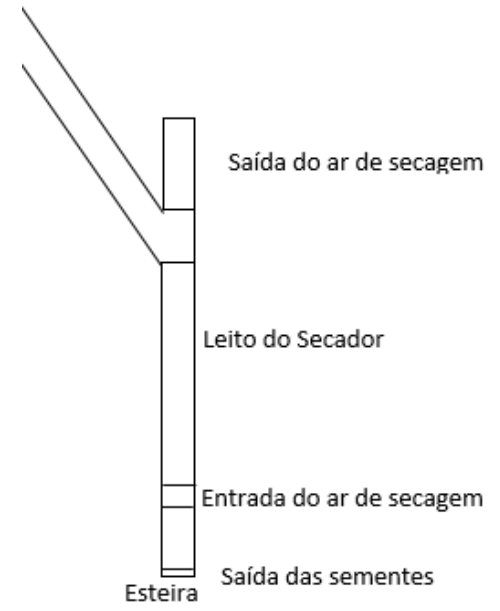

As equações e parâmetros utilizados para a fase secundária estão descritos na Tabela 1.

Tabela 1 - Parâmetros da fase secundária sementes de soja.

\begin{tabular}{|c|c|c|}
\hline \multicolumn{3}{|c|}{ Fase Secundária } \\
\hline Diâmetro (m) & constante & 0,006 \\
\hline $\begin{array}{l}\text { Viscosidade granular } \\
\left(\mathrm{kg} \mathrm{m}^{-1} \mathrm{~s}^{-1}\right)\end{array}$ & $\begin{array}{c}\text { Syamlal- } \\
\text { obrien }\end{array}$ & \\
\hline $\begin{array}{l}\text { Viscosidade granular bulk } \\
\left(\mathrm{kg} \mathrm{m}^{-1} \mathrm{~s}^{-1}\right)\end{array}$ & Lun-et-al & - \\
\hline $\begin{array}{l}\text { Viscosidade friccional } \\
\left(\mathrm{kg} \mathrm{m}^{-1} \mathrm{~s}^{-1}\right)\end{array}$ & Schaeffer & - \\
\hline $\begin{array}{l}\text { Ângulo de fricção interna } \\
\left({ }^{\circ}\right)\end{array}$ & constante & 30,000 \\
\hline Pressão friccional $(\mathrm{Pa})$ & $\begin{array}{l}\text { Based- } \\
\text { ktgf }\end{array}$ & - \\
\hline Módulo friccional $(\mathrm{Pa})$ & Derived & - \\
\hline $\begin{array}{l}\text { Limite de empacotamento } \\
\text { friccional }\end{array}$ & constante & 0,61 \\
\hline $\begin{array}{l}\text { Temperatura granular } \\
\left(\mathrm{m}^{2} \mathrm{~s}^{-2}\right)\end{array}$ & Algebraic & - \\
\hline Pressão dos sólidos $(\mathrm{Pa})$ & Lun-et-al & - \\
\hline Distribuição radial & Lun-et-al & - \\
\hline $\begin{array}{l}\text { Módulo de elasticidade } \\
(\mathrm{Pa})\end{array}$ & Derived & - \\
\hline Limite de empacotamento & constante & 0,61 \\
\hline
\end{tabular}

Para a análise dos resultados dos perfis de velocidade e porosidade do leito foram criadas linhas ao longo do comprimento do secador, conforme apresentado na Figura 2.

Figura 2 - Esquema do secador de leito deslizante com escoamento contracorrente com descrição das linhas de análise.

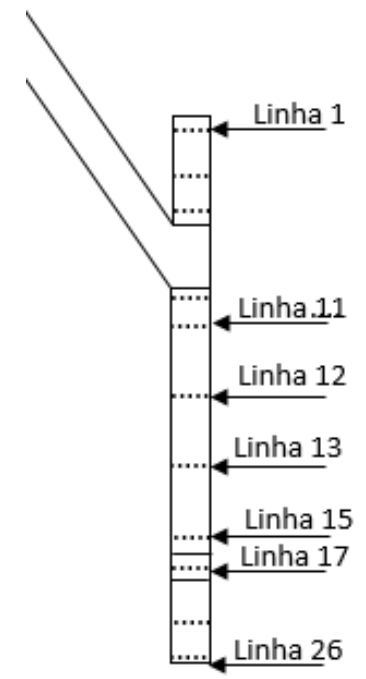

Para o estudo do perfil de porosidade e velocidade, foram analisados os resultados das simulações para o tempo de 10 segundos, com velocidade de entrada do ar no secador igual a $1,42 \mathrm{~m} \cdot \mathrm{s}^{-1}$, variando o coeficiente de especularidade de 0 a 1.

Foi realizada a comparação entre os resultados de simulação neste trabalho e os dados experimentais obtidos por Souza (2011), no qual a autora coletou dados na saída do ar de secagem, para duas situações: célula de secagem vazia e preenchida por sólidos em escoamento; na qual a última situação foi objeto de comparação neste estudo.

\section{RESULTADOS E DISCUSSÃO}

Os resultados da simulação do teste para o perfil de porosidade e de velocidade, variando o coeficiente de especularidade, são apresentados nas Figuras 3 a 6 para algumas 
posições de análise ao longo do comprimento do secador.

As Figuras 3 e 4 apresentam os perfis de porosidade das linhas 13 e 11 , respectivamente, para o teste com velocidade do ar igual a $1,42 \mathrm{~m} \cdot \mathrm{s}^{-1}$. Pode ser observado, que para a linha de análise 13, que se encontra na região superior da entrada de ar do secador, há uma variação do perfil de porosidade com a aproximação da parede do secador.

Verifica-se também, um efeito relacionado ao coeficiente de especularidade; para a simulação sem o coeficiente $(=0)$, há um efeito mais acentuado no perfil de porosidade, apesar da variação ser da ordem de $0,01(1,67 \%)$; e, que, com o aumento do coeficiente de especularidade o perfil se aproxima do perfil de porosidade sem este coeficiente; o perfil se torna amortecido na região central do leito.

Para a linha 11, que se encontra próxima a entrada de soja no secador observase efeito similar aos da linha 13, porém com menor variação de acordo com o coeficiente de especularidade.

Figura 3 - Perfis de porosidade da linha 13 (Coeficiente de especularidade: $\diamond 0,0 ; \square 0,1 ; \Delta 0,2 ; \square 0,4 ; * 0,6$; $\bullet 0,8 ; \boldsymbol{\Delta} 1,0)$.

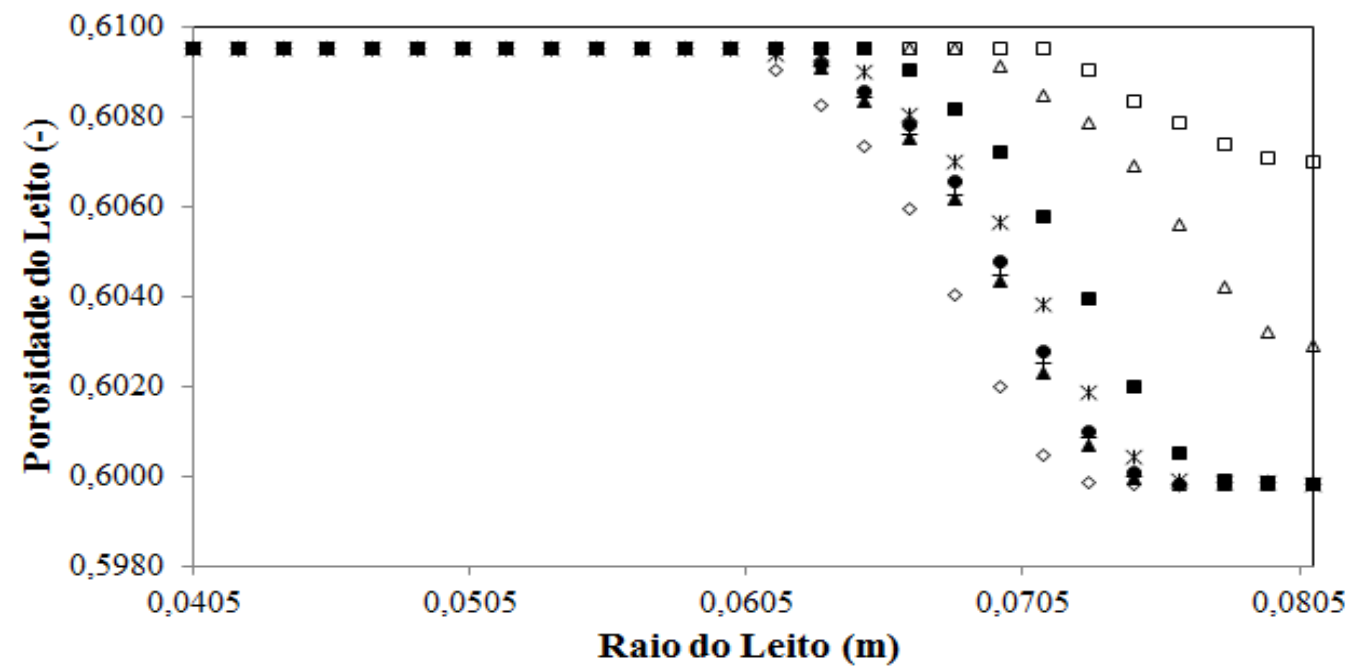

Figura 4 - Perfis de porosidade da linha 11 (Coeficiente de especularidade: $\diamond 0,0 ; \square 0,1 ; \Delta 0,2 ; \square 0,4 ; * 0,6$; $\bullet 0,8 ; \boldsymbol{\Delta} 1,0)$.

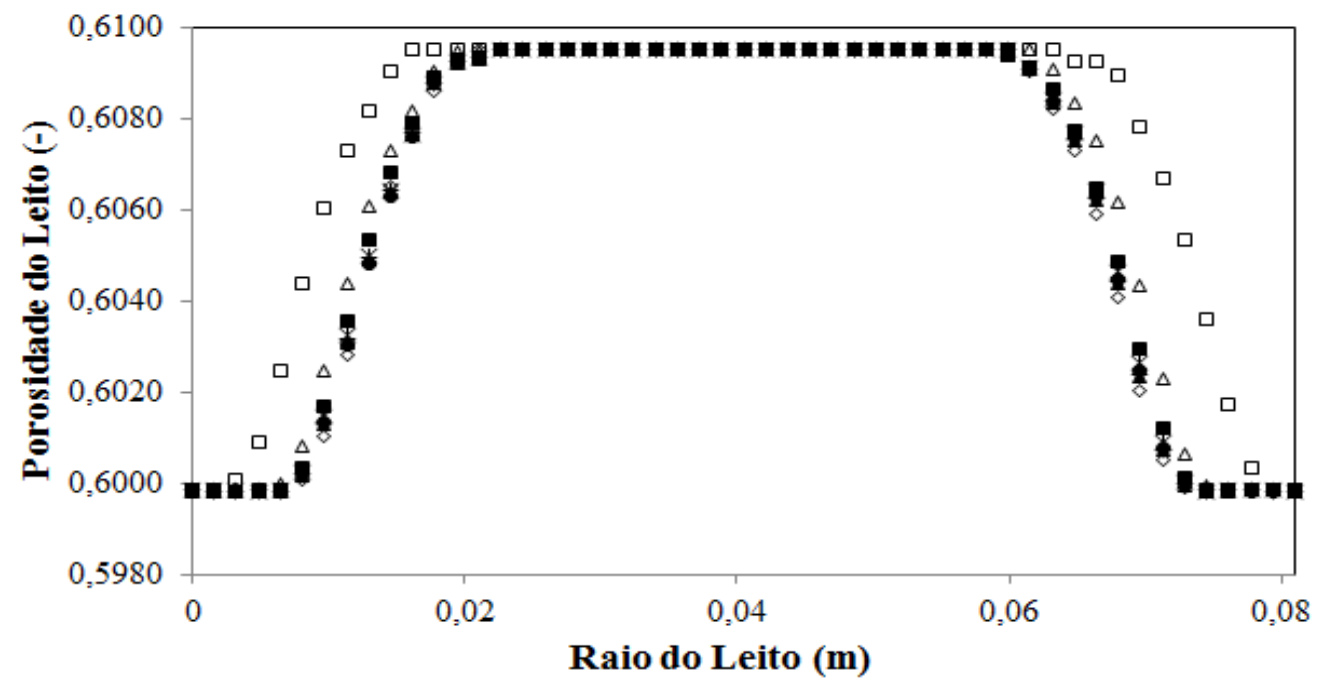


As Figuras 5 e 6 apresentam, os perfis de velocidade do ar para as linhas 13 e 2, respectivamente, com velocidade do ar de entrada igual a 1,42 m.s ${ }^{-1}$. Na Figura 5, observa-se a mesma variação em relação ao coeficiente de especularidade, conforme discutido anteriormente. $\mathrm{Na}$ figura 6 , de acordo com a linha de análise 2, próxima à saída do secador, o perfil de velocidade com coeficiente de especularidade igual a 0,6 apresentou melhor ajuste aos dados experimentais. Esta linha de análise se assemelha à condição de coleta dos dados experimentais feitos por Souza (2001).

Figura 5 - Perfis de velocidade do ar da linha 13 (Coeficiente de especularidade: $\diamond 0,0 ; \square 0,1 ; \Delta 0,2 ; \square 0,4 ; *$ $0,6 ; \bullet 0,8 ; \boldsymbol{\Delta} 1,0)$.

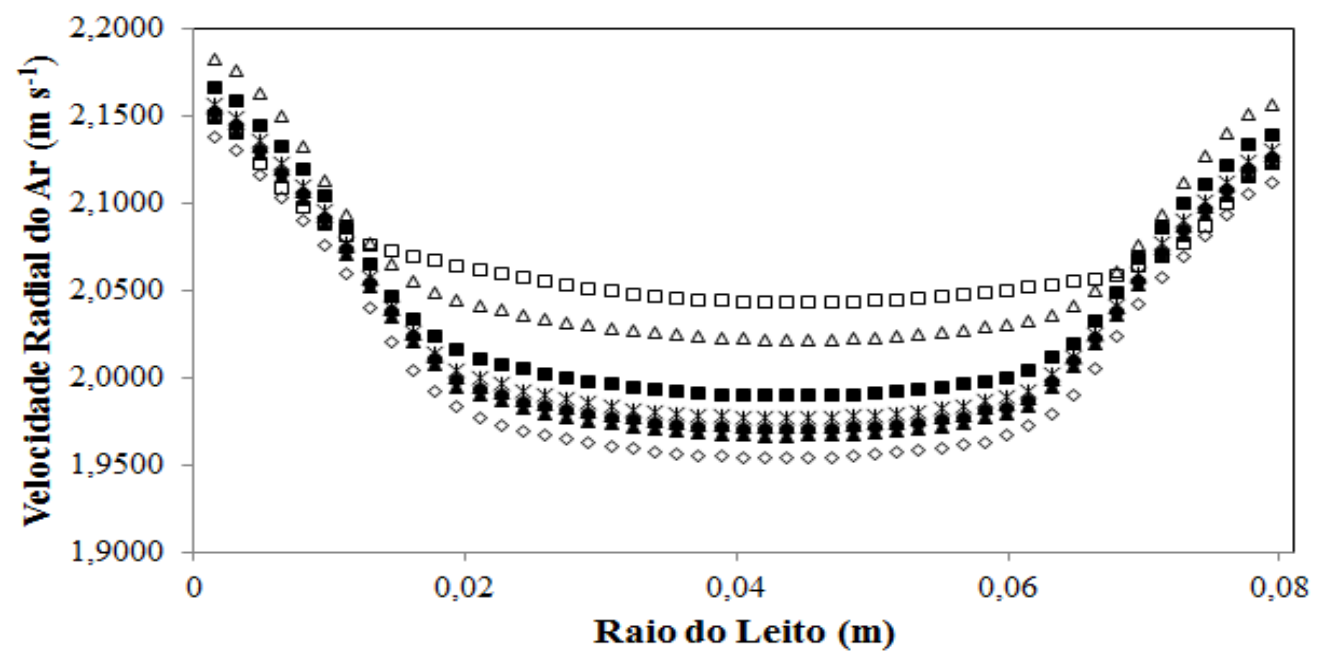

Figura 6 - Perfis de velocidade do ar da linha 2 (Coeficiente de especularidade: * 0,6; • 0,8; + 0,9; x Experimental).

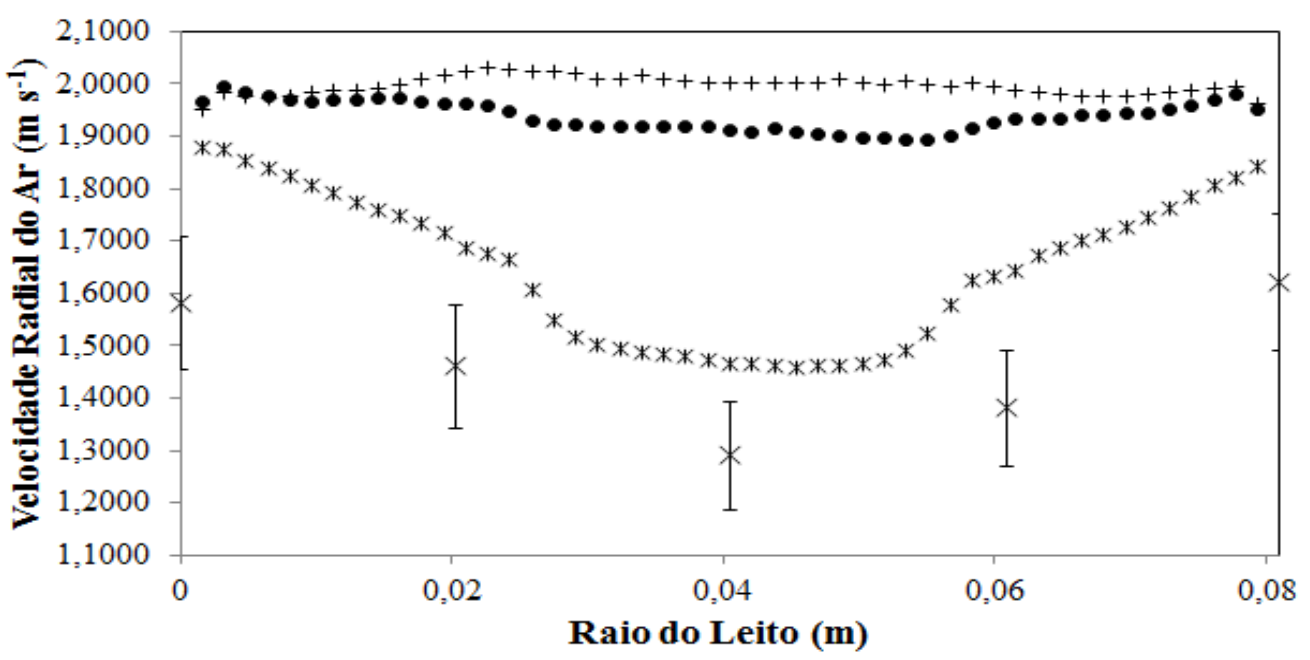

A variação do coeficiente de especularidade, que é uma medida da fração de colisões que transferem movimento à parede, influenciou nos perfis de porosidade e de velocidade, contribuindo para uma melhor adequação aos dados experimentais nos testes realizados. Os resultados demonstraram que para a situação em que a célula encontra-se com sólidos em escoamento, as regiões próximas à parede do secador apresentam níveis superiores de velocidade.

Os perfis apresentados para o tempo de simulação de $10 \mathrm{~s}$ condizem com a observação de que os valores atingiram o 
estado estacionário, sendo portanto, correto comparar os dados experimentais com os dados aqui simulados. Desta forma, foi observado o fato de que há uma variação no perfil de porosidade e velocidade do ar na secagem em leito deslizante.

\section{CONCLUSÃO}

A fluidodinâmica computacional mostrou-se de grande ajuda para a verificação do efeito de parede nos perfis de porosidade e velocidade do fluido, confirmando que a suposição de perfil plano de velocidade da fase fluida, no modelo a duas fases, não é válida na velocidade do ar analisada.

\section{REFERÊNCIAS}

BARROZO, M. Transferência de calor e massa entre o ar e sementes de soja em leito deslizante e escoamento. Tese (Doutorado) - Faculdade de Engenharia Química, Universidade Federal de São Carlos, São Carlos, 1995. P. 163.

BÉTTEGA, R.; CORRÊA, R. G.; FREIRE, J. Velocity profile in fixed beds: a study on the representativeness of the experimental measurement of downstream flow characteristics. Drying Technology Journal, v. 25, n. 7 , p. $1175-1183,2007$.

DUARTE, C. Estudo experimental e de simulação da fluidodinâmica e recobrimento em leito de jorro. Tese (Doutorado) — Faculdade de Engenharia Química, Universidade Federal de Uberlândia, 2006.

FAHIEN, R.; STANKOVICH, I. An equation for the velocity profile in packed columns. Chemical Engineering Science, v. 34, p. 1350-1354, 1979.

FLUENT. User's guide. 2006.
LIRA, T.; BARROZO, M.; ASSIS, A. Concurrent moving bed dryer modelling: Sensitivity of physicochemical parameters and influence of air velocity profiles. Applied Thermal Eng., v. 29, p. 892-897, 2009.

LIRA, T.; BARROZO, M.; ASSIS, A. Drying of soybean seeds in a countercurrent moving bed: The effect of the radial air profile. 15th International Drying Symposium - IDS, 2006.

PFEIFER, A. A. Análise da Modelagem, Simulação e Otimização da Secagem em Leito Deslizante com Escoamentos Paralelos. Tese (Doutorado) - UFU, Universidade Federal de Uberlândia, 2011. Uberlândia - MG.

SOUZA, A. Estudo fluidodinâmico e de transferência de calor e massa em leito deslizante contracorrente. Dissertação (Mestrado) - Faculdade de Engenharia Química, Universidade Federal de Uberlândia, Uberlândia, 2001.

VALENÇA, G.; MASSARANI, G. Avaliação de secadores em fluxo concorrentes e contracorrentes. Anais do XXIV ENEMP, v. 1, p. 225-230, 1996. Uberlândia, MG.

VORTMEYER, D.; SCHUSTER, J. Evaluation of steady flow profiles in rectangular and circular packed beds by a variational method. Chemical Engineering Science, v. 38, p. 1691-1699, 1983.

\section{AGRADECIMENTOS}

Os autores agradecem à FAPEMIG pela bolsa de Iniciação Científica. 